\title{
Clinicopathologic Correlation: Firm Mass on the Lateral Aspect of the Posterior Maxilla
}

\author{
Darin T. Johnston, James J. Closmann, Pallavi Parashar, Thomas J. Borris \\ Oral and Maxillofacial Surgery, University of Colorado School of Dental Medicine, Aurora, USA \\ Email: darin.johnston@ucdenver.edu
}

Received 27 February 2014; revised 31 March 2014; accepted 8 April 2014

Copyright (C) 2014 by authors and Scientific Research Publishing Inc.

This work is licensed under the Creative Commons Attribution International License (CC BY). http://creativecommons.org/licenses/by/4.0/

c) (i) Open Access

\begin{abstract}
We present the case of a 45-year-old Hispanic female with a slowly enlarging mass on her left maxilla in the region of teeth \#15 and \#16. A differential diagnosis is presented along with the histologic findings and treatment rendered. The prognosis and biology of the lesion are discussed.
\end{abstract}

\section{Keywords}

\section{Exostosis, Gingival Diseases, Oral Pathology, Pyogenic Granuloma, Surgery}

\section{Introduction}

A 40-year-old Hispanic female was referred to the oral and maxillofacial surgery clinic of the University of Colorado School of Dental Medicine for severe pain in the upper left quadrant, which presented for several weeks. The patient had a non-contributory medical history and was not taking any medications, was a nonsmoker and consumed social alcohol only.

On panoramic radiographic examination, the patient was noted to have a large carious lesion on tooth \#15 (Figure 1). Teeth \#15 and \#16 were both noted to be mobile and extremely tender to percussion and palpation. Of note, the patient was observed to have a large, approximately $2 \mathrm{~cm}$ in greatest dimension, pinkish mucosal covered mass on the lateral (buccal) aspect of her posterior maxilla over the area of teeth \#15 and \#16 (Figure 2). When questioned about the mass in her maxilla, the patient stated it had been there for many years and was told it was bone by her previous dentist. The mass was firm, non-tender, showed very slight mobility and no bleeding was noted.

Teeth \#15 and \#16 were recommended for extraction by the patient's restorative dentist due to caries and periodontal disease. A biopsy of the soft tissue on the lateral aspect of her maxilla in the same region was recommended. The patient consented to the recommended extractions and biopsy.

A differential diagnosis of the maxillary lesion was formulated and included: maxillary exostosis, peripheral 


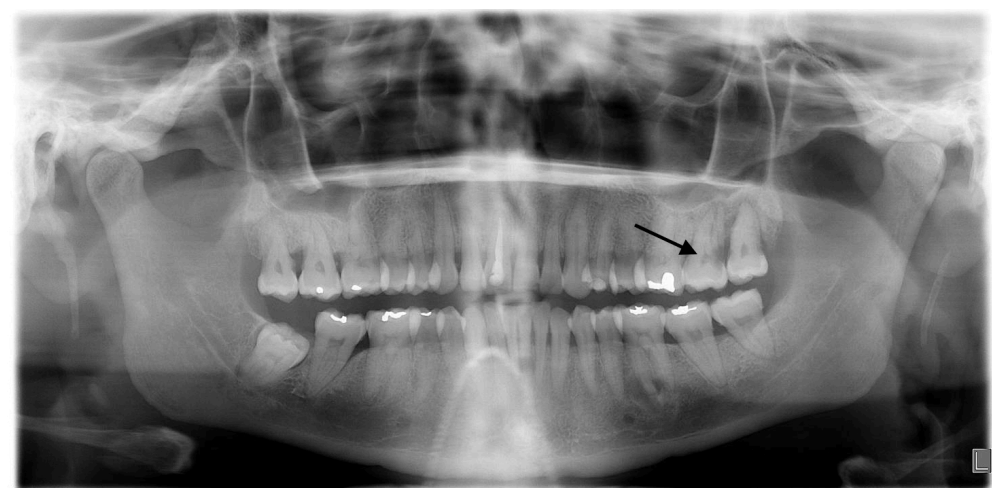

Figure 1. Panoramic radiograph taken at initial visit demonstrating severe caries on tooth \#15 (arrow).

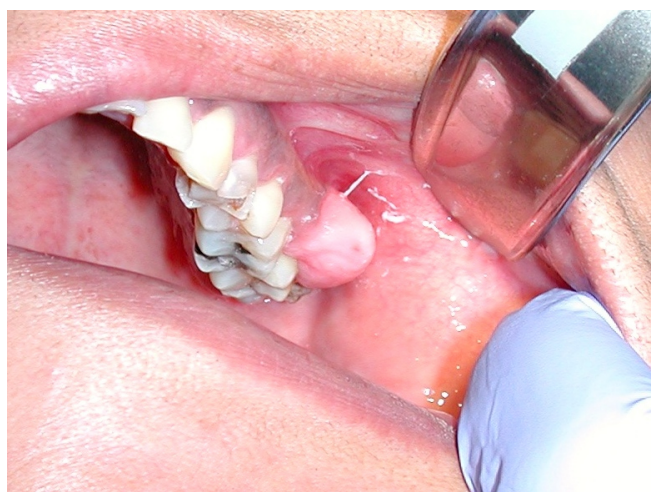

Figure 2. Clinical photograph taken at initial visit showing pinkish mucosal covered mass on lateral aspect of left posterior maxilla.

ossifying fibroma, peripheral fibroma, peripheral giant cell granuloma, and possibly a peripheral manifestation of an odontogenic tumor.

\section{Methods}

Using local anesthesia only the procedures were performed without complication. The teeth were removed easily with forceps and elevator technique. An excisional biopsy was performed on the lateral maxillary mass using a \#15 blade. Care was taken to remove the lesion with a cuff of healthy appearing tissue at the base (Figure 3). The specimen was submitted for histologic examination in formalin.

\section{Results}

The biopsy sample represented a mucosal nodule covered by intact keratinizing squamous epithelium (Figure 4). The epithelium was supported by connective tissue, which was rich in collagen. Within the collagen, there was evidence of ossifications representing a mineralized product such as bone (Figure 5). Based on these histologic findings, a diagnosis of peripheral ossifying fibroma was rendered.

The patient was scheduled to return to the clinic in approximately one week to review the pathology report and determine if any further treatment was necessary. The histologic diagnosis was returned "peripheral ossifying fibroma". The patient was informed and no further treatment for the lesion was scheduled. The patient was then referred to her general dentist for definitive care of her oral health needs.

\section{Discussion}

Peripheral ossifying fibroma (POF) is a reactive non-neoplastic lesion, and is not a soft tissue version of the 


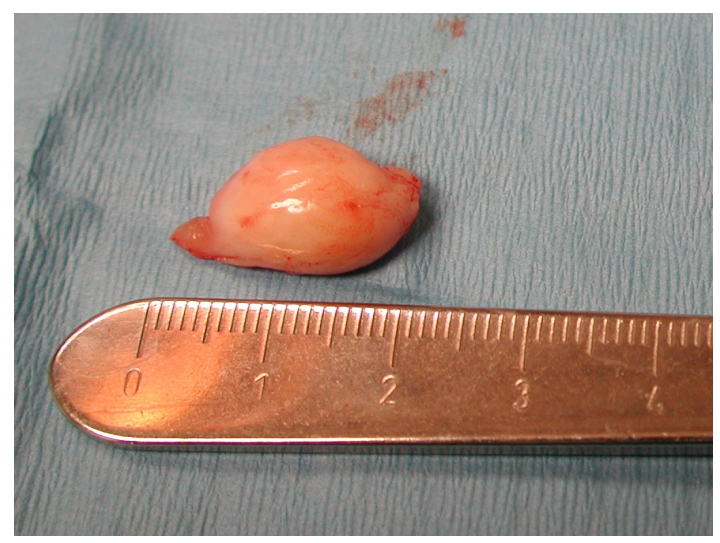

Figure 3. Clinical photograph taken post-operatively showing excised mass.

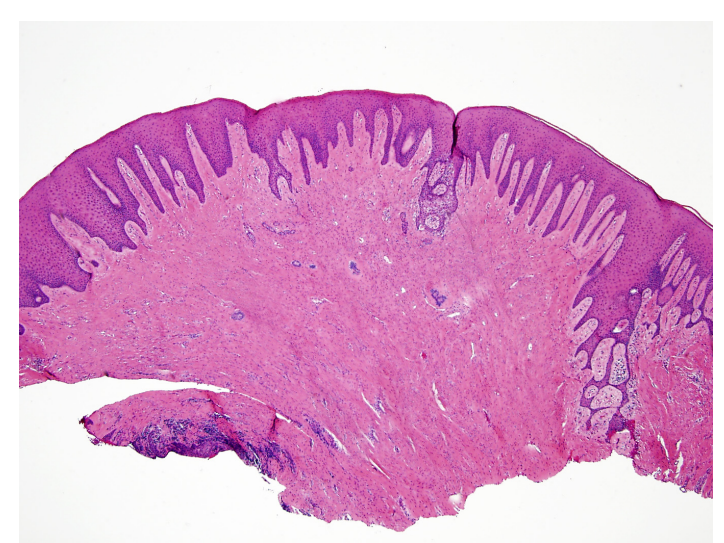

Figure 4. H\&E stained biopsy specimen at $40 \times$ power representing a mucosal nodule covered by intact keratinizing squamous epithelium.

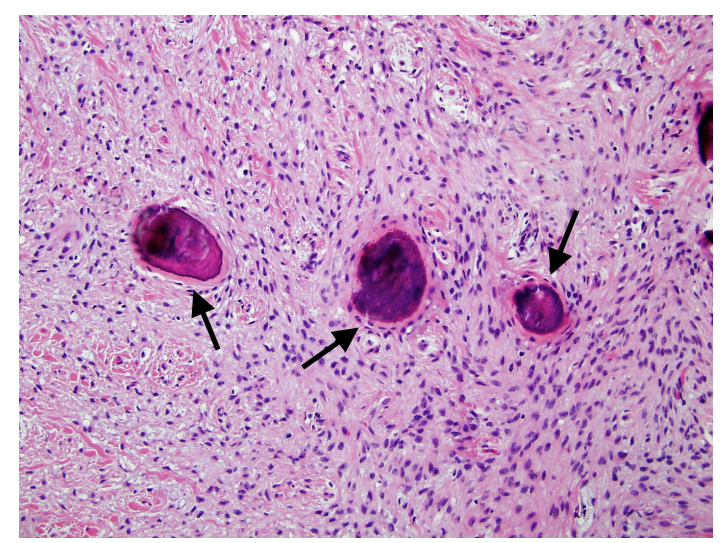

Figure 5. H\&E stained biopsy specimen at 400× power representing a mineralized product such as bone in the connective tissue (arrows).

central ossifying fibroma. It presents on the gingiva as a painless, firm, localized growth with either a sessile or pedunculated base which does not blanch when palpated [1]-[4]. The surface can be similar to adjacent unaffected gingiva or red, and can be ulcerated [5]. The ulcerated type likely represents a POF of 3 - 6 months duration, whereas the non-ulcerated type is of 12 - 24 months duration [1]. These different stages can be misdiag- 
Table 1. Summary of data reported by Buchner et al. regarding the four most common gingival hyperplastic lesions [2].

\begin{tabular}{ccccc}
\hline Diagnosis & Frequency & Location & Gender & Age \\
\hline FFH & $32 \%$ & Mx 48\% Mn 52\% & F 57\% M 43\% & $20-60$ \\
PG & $29 \%$ & Mx 58\% Mn 42\% & F 69\% M 31\% & $20-60$ \\
POF & $20 \%$ & Mx 56\% Mn 44\% & F 61\% M 39\% & $10-40$ \\
PGCG & $19 \%$ & Mx 45\% Mn 55\% & F 48\% M 52\% & $40-60$ \\
\hline
\end{tabular}

nosed and incorrectly treated with superficial excision. The ulcerated type can be mistaken for a pyogenic granuloma, and the non-ulcerated for an irritation fibroma or epulis [4].

The interdental papilla is the most common site, however there are reports of lesions adjacent to teeth in an edentulous area [1]. The lesion occurs more commonly in women, and occurs almost equally on the maxilla or mandible [1] [5]. While reports exist of POF with diameters reaching $9 \mathrm{~cm}$ which displace adjacent teeth, lesions are most commonly $<2 \mathrm{~cm}$ [1] [3]. There is often radiographic evidence of bone and other calcifications [5].

Similar to other gingival swellings, POF most commonly develops in reaction to irritation. Such sources could include chronic local irritation or trauma from calculus, food impaction, restorations that have irregular margins, low-grade trauma, and iatrogenic factors [2] [6]. POF originates from the periodontal ligament and/or periosteum which is capable of producing the bone, cementum, and fibrous tissue [1].

A differential diagnosis of a solitary gingival mass, listed in order of decreasing frequency, should include: focal fibrous hyperplasia (FFH), pyogenic granuloma (PG), peripheral ossifying fibroma (POF), peripheral giant cell granuloma (PGCG) (Table 1).

The anterior maxilla is the most common site of FFH, PG, and POF. The posterior mandible is the most common site of PGCG. It is worth noting that the difference in maxillary and mandibular presentations of the four lesions is nominal. Furthermore, only PG and POF demonstrate a significant difference in gender, with each demonstrating a female predilection of approximately 2:1. FFH and PG are most commonly found in the 3rd 7th decade, POF 2nd - 3rd decade, and PGCG 5th - 7th decade [2] [4]. Interestingly, Kenney speculated a potential hormonal role in cases of POF [5].

Recurrence of the lesion occurs between $8 \%$ - 30\% of the time [1] [4] [6]. To minimize recurrence, treatment is complete excision (with 2 mm margins) and possible extraction of involved teeth should include the periodontal ligament. Some advocate that all affected soft tissue should be excised down to bone [4].

A gingival hyperplastic lesion that indicates a source of irritation is present, which might have otherwise been unrecognized. In this case, a patient presented with a gingival mass that had previously been incorrectly determined to be an exostosis. Several key characteristics can guide the dentist when evaluating a gingival nodule or mass. These include the consistency, location, surface, and radiographic appearance of the mass. An accurate evaluation enables the dentist to develop a differential diagnosis and treatment plan which best serves the patient.

\section{References}

[1] Buchner, A. and Hansen, L.S. (1987) The Histomorphologic Spectrum of Peripheral Ossifying Fibroma. Oral Surgery, Oral Medicine, Oral Pathology, 63, 452-461. http://dx.doi.org/10.1016/0030-4220(87)90258-1

[2] Buchner, A., Shnaiderman-Shapiro, A. and Vered, M. (2010) Relative Frequency of Localized Reactive Hyperplastic Lesions of the Gingiva: A Retrospective Study of 1675 Cases from Israel. Journal of Oral Pathology \& Medicine, 39, 631-638. http://dx.doi.org/10.1111/j.1600-0714.2010.00895.x

[3] Childers, E.L.B., Morton, I., Fryer, C.E. and Shokrani, B. (2013) Giant Peripheral Ossifying Fibroma: A Case Report and Clinicopathologic Review of 10 Cases from the Literature. Head and Neck Pathology, 7, 356-360. http://dx.doi.org/10.1007/s12105-013-0452-1

[4] Walters, J.D., Will, J.K., Hatfield, R.D., Cacchillo, D.A. and Raabe, D.A. (2001) Case Report Excision and Repair of the Peripheral Ossifying Fibroma: A Report of 3 Cases. Journal of Periodontology, 72, 939-944. http://dx.doi.org/10.1902/jop.2001.72.7.939

[5] Kenney, J.N., Kaugars, G.E. and Abbey, L.M. (1989) Comparison between the Peripheral Odontogenic Fibroma. Journal of Oral and Maxillofacial Surgery, 47, 378-382. http://dx.doi.org/10.1016/0278-2391(89)90339-X 
[6] Shumway, B.S., Eskan, M.A. and Bernstein, M.L. (2013) Recurrent Gingival Fibrous Lesions: Comparison of 2 Cases and Potential Need for Additional Classification. Oral Surgery, Oral Medicine, Oral Pathology, Oral Radiology, 116, e287-e296. http://dx.doi.org/10.1016/j.0000.2013.02.004 